\title{
Apendicitis poscolonoscopia: reporte de caso y revisión de la literatura
}

\section{Post-colonoscopy appendicitis: Case report and literature review}

\author{
Camilo Ramírez Giraldo, MD, ${ }^{1}$ Ovidio Fernando Molano Chavarría, MD, ${ }^{2}$ Juan Guillermo Holguín Henao, MD, ${ }^{3 *}$ \\ Carlos Edgar Figueroa Avendaño, MD. ${ }^{4}$
}

\footnotetext{
1 Médico cirujano, epidemiólogo, residente de cirugía general. Universidad del Rosario y Hospital Universitario Mayor. Bogotá, Colombia.

2 Médico cirujano, residente de cirugía general. Universidad del Rosario y Hospital Universitario Mayor. Bogotá, Colombia.

3 Médico cirujano, Fellowship en Coloproctología. Universidad del Rosario y Hospital Universitario Mayor. Hospital Universitario Barrios Unidos. Bogotá, Colombia.

${ }_{4}^{4}$ Cirujano coloproctólogo, Jefe del servicio de Coloproctología, Hospital Universitario Mayor Méderi. Bogotá, Colombia.

*Correspondencia: Juan Guillermo Holguín Henao, jgh3000@gmail.com
}

Fecha recibido: $\quad 03 / 12 / 18$ Fecha aceptado: 20/01/19

\begin{abstract}
Resumen
Presentamos el caso de una paciente de 83 años sin antecedentes de importancia, a quien se le realizó una colonoscopia de tamización $3 \mathrm{~h}$ antes del inicio de la sintomatología. La paciente consultó al servicio de urgencias por presentar dolor en la fosa ilíaca derecha de $12 \mathrm{~h}$ de evolución. En efecto, durante el examen físico, se encontró un dolor localizado en la fosa ilíaca derecha.

Ante la sospecha de una complicación relacionada con la colonoscopia, se ordenó una tomografía de abdomen, la cual mostró signos tomográficos de apendicitis. Se realizó entonces una apendicetomía por laparoscopia, sin complicaciones, pero se evidenció un apéndice cecal perforado.
\end{abstract}

\section{Palabras clave}

Colonoscopia, apendicitis, complicación.

\section{Abstract}

We present the case of an 83-year-old patient who had no significant medical history. A screening colonoscopy had been performed three 3 hours before onset of pain in the right iliac fossa. Twelve hours later, the patient to the emergency department. Physicians suspected that the pain was a complication related to colonoscopy and ordered an abdominal CT scan which showed tomographic signs of appendicitis. A laparoscopic appendectomy was performed and removed a perforated cecal appendix without complications.

\section{Keywords}

Colonoscopy, appendicitis, complication.

\section{INTRODUCCIÓN}

En la práctica médica, una complicación es el resultado desfavorable de un procedimiento diagnóstico o terapéutico. Mientras una intervención se realiza con el fin de obtener un beneficio, esta puede causar algún tipo de daño. Por tanto, antes de realizar un procedimiento siempre debemos calcular la probabilidad de complicaciones y hacer un balance contra los posibles beneficios (1).

La colonoscopia es un procedimiento que se realiza de forma rutinaria en los servicios de coloproctología y de gas- troenterología a nivel mundial. Este es el procedimiento de elección para la tamización del cáncer colorrectal y se usa para el diagnóstico y el tratamiento de otras patologías de la mucosa colorrectal.

Sin embargo, no es un procedimiento inocuo y se pueden presentar complicaciones como perforación, sangrado y lesión esplénica, y otras menos frecuentes como priapismo, obstrucción intestinal, isquemia mesentérica, vólvulo del ciego, pancreatitis, perforaciones del intestino delgado y apendicitis (1).

En efecto, la apendicitis poscolonoscopia es una complicación muy rara, con una incidencia descrita de aproxi- 
madamente $0,038 \%$. No obstante, la incidencia es difícil de establecer, dado que solo existen reportes de caso. De hecho, en la literatura se han descrito, hasta 2017, 41 casos, de los cuales solo uno se desarrolló en Latinoamérica $(2,3)$.

\section{CASO CLÍNICO}

Mujer de 83 años sin antecedentes patológicos de interés, a quien se le realizó una colonoscopia diagnóstica y se encontraron múltiples divertículos en el colon sigmoide. La exploración completa tuvo una duración aproximada de 12 min y no se hallaron alteraciones en el ciego, así como tampoco en el orificio apendicular. Además, no se observaron otras alteraciones en el resto del colon (Figura 1). Por tanto, se le dio egreso a la paciente, sin que se evidenciara algún tipo de complicación inmediata.

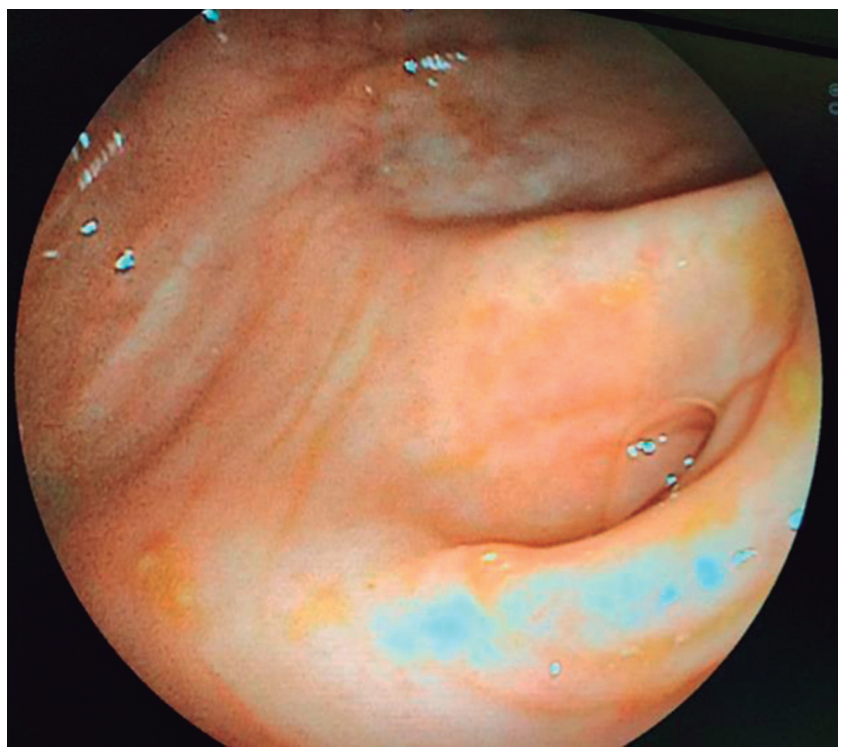

Figura 1. Orificio apendicular sano en la colonoscopia.

Después de $12 \mathrm{~h}$ del procedimiento, la paciente decidió consultar al servicio de urgencias, por causa de un cuadro de dolor abdominal en aumento, localizado en la fosa ilíaca derecha, el cual inició luego de $3 \mathrm{~h}$ de haber ocurrido el egreso. Durante el examen físico, llamó la atención el dolor localizado en la fosa ilíaca derecha con defensa abdominal.

Por lo tanto, se realizaron paraclínicos con evidencia de leucocitosis $\left(20,6 \times 10^{9} \mathrm{Cel} / \mathrm{L}\right)$ y neutrofilia $(90 \%)$. Debido a la sospecha de una perforación causada por el procedimiento realizado, se decidió practicar una tomografía de abdomen (Figura 2). En ella se observó un apéndice retrocecal de $11 \mathrm{~mm}$, con alteración de la grasa adyacente, razón por la cual se efectuó una apendicetomía por laparoscopia.

El procedimiento quirúrgico se realizó sin complicaciones y permitió encontrar un apéndice cecal perforado, con escaso líquido de reacción peritoneal en la fosa ilíaca derecha. El paciente cursó con un posoperatorio sin complicaciones y al tercer día se definió su egreso hospitalario. Entre tanto, el estudio de patología describió una apendicitis aguda perforada y una periapendicitis.

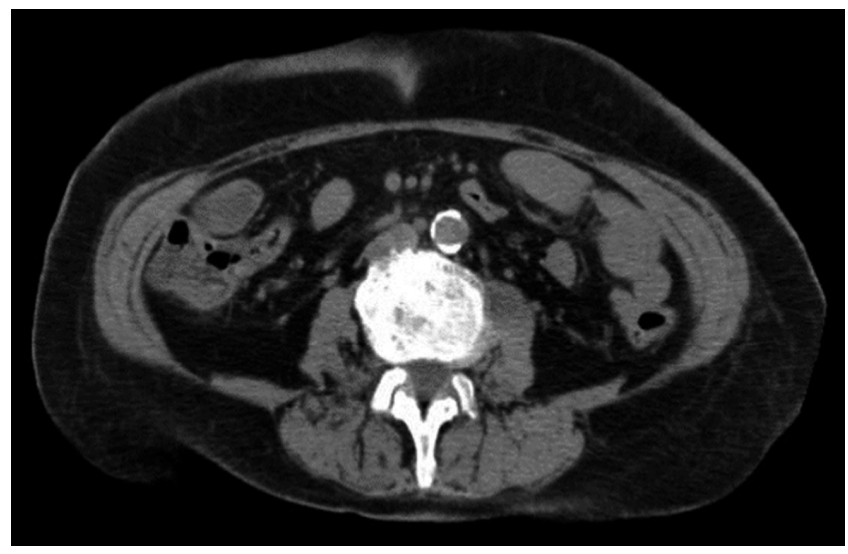

Figura 2. Tomografía de abdomen con cambios inflamatorios del apéndice cecal.

\section{DISCUSIÓN}

La apendicitis aguda poscolonoscopia es una rara complicación, descrita por primera vez en 1988 por Hougton y Aston (4). Después de su hallazgo, se han reportado 41 casos y solo uno de ellos fue reseñado en la literatura latinoamericana. Este constituye un diagnóstico de dificultad, debido a que el dolor puede ser secundario a un gas retenido o a un espasmo colónico secundario a la colonoscopia.

Además, la apendicitis aguda poscolonoscopia debe diferenciarse del síndrome pospolipectomía, el cual se presenta con una clínica similar de dolor, irritación peritoneal, leucocitosis y fiebre (3).

De los casos reportados en la literatura, la mayoría son hombres con una mediana de edad de 53,9 años; el más longevo es de 84 años. Entre tanto, el tiempo entre la colonoscopia y la aparición de la sintomatología fue variable, pero en la mayoría de los escenarios (28 pacientes) ocurrió durante las primeras $24 \mathrm{~h}$, al igual que en el caso descrito.

Dentro de los casos analizados, 4 recibieron un tratamiento conservador, mientras que en 5 de ellos no fue especificado el manejo. El resto de los pacientes tuvieron un abordaje quirúrgico, tal y como sucedió en nuestro caso. Entre los hallazgos intraoperatorios, se encontraron 14 apéndices perforados (como en nuestra paciente) $(5,6)$, lo cual puede relacionarse con el mecanismo de lesión que ocurre durante la colonoscopia (7).

La tomografía de abdomen se constituye en el estudio de elección para obtener un diagnóstico preoperatorio, en vista de que, como ya se refirió, la causa del dolor puede 
tener orígenes no quirúrgicos o estar relacionada con complicaciones más frecuentes. Por tanto, la tomografía de abdomen contribuye a establecer el manejo definitivo según los hallazgos.

Dentro de la causalidad de la apendicitis aguda poscolonoscopia se han descrito múltiples teorías, como la existencia de una patología subclínica del apéndice, el barotrauma, la impactación fecal, la intubación de la luz apendicular o la lesión de la mucosa alrededor del orificio apendicular $(3,8)$.

Si bien podría considerarse que el desarrollo de una apendicitis poscolonoscopia es coincidencia, quizás el principio de la navaja de Ockham sugiera que, en efecto, existe una relación causal. Esta cuestión no se ha dilucidado por completo (9). De ahí surge el interrogante: ¿coincidencia o causalidad?

\section{CONCLUSIONES}

La apendicitis poscolonoscopia es una complicación poco frecuente y tiene una incidencia verdadera desconocida, dado el bajo número de casos reportados a nivel mundial. No obstante, esta afección siempre debe ser incluida en el diagnóstico diferencial de los pacientes con dolor abdominal posterior a una colonoscopia. Un diagnóstico oportuno es la piedra angular para evitar complicaciones.

\section{REFERENCIAS}

1. Church J. Complications of colonoscopy. Gastroenterol Clin North Am. 2013;42(3):639-57. http://dx.doi. org/10.1016/j.gtc.2013.05.003

2. Loureiro M, Bonin E, Leiner C, Weigmann SC, Fontana A. Apendicite aguda pós colonoscopia: desafio diagnóstico e tratamento minimamente invasivo - relato de caso. Rev Col Bras Cir. 2011;38(5):365-368. https://doi.org/10.1590/ S0100-69912011000500015

3. Chae HS, Jeon SY, Nam WS, Kim HK, Kim JS, Kim JS, et al. Acute appendicitis caused by colonoscopy. Korean J Intern Med. 2007;22(4):308-311. https://doi.org/10.3904/ kjim.2007.22.4.308

4. Houghton A, Aston N. Appendicitis complicating colonoscopy. Gastrointest Endosc. 1988;34(6):489. https://doi. org/10.1016/S0016-5107(88)71451-0

5. Paramythiotis D, Kofina K, Papadopoulos V, Michalopoulos A. Diagnostic Colonoscopy Leading to Perforated Appendicitis: A Case Report and Systematic Literature Review. Case Rep Gastrointest Med. 2016;2016:1378046. https://doi.org/10.1155/2016/1378046

6. Sanz M, Labarta M, Cuartero B, Mejía D. Apendicitis: complicación de una colonoscopia. Hospi Clínico Univ Miguel Servet. 2011:2-6.

7. Zhou XC, Huang CW, Dai YY, Huang ZY, Lou Z. Perforated appendicitis after colonoscopy: cause or coincidence? A rare case report and literature review. Medicine (Baltimore). 2017;96(46):e8747. https://doi.org/10.1097/ MD.0000000000008747

8. Rodríguez-Otero Luppi C, Salas Muñoz J, Targarona EM, Rodríguez Blanco M, Bollo J, Trias M. Acute appendicitis after colonoscopy: coincidence or cause? Gastroenterol Hepatol. 2011;34(7):516-7. https://doi.org/10.1016/j. gastrohep.2011.04.004

9. Shaw D, Gallardo G, Basson MD. Post-colonoscopy appendicitis: A case report and systematic review. World J Gastrointest Surg. 2013;5(10):259-263. https://doi. org/10.4240/wjgs.v5.i10.259 